\title{
Relationship between the monoamine oxidase gene overactivity and the other pathophysiological and behavioral parameters implicated in memory deficiency in albino Winstar rats as Alzheimer's disease model
}

Halla Abdul-Hadi Chabuk*

Department of Biology, College of Science, University of Babylon, Babil 51001, Iraq

Zahraa Isam Jameel

Department of Biology, College of Science, University of Babylon, Babil 51001, Iraq

*Corresponding author. Email: sci.halah.a.hadi@uobabylon.edu.iq

\section{Article Info}

https://doi.org/10.31018/

jans.v13i4.3024

Received: September 21, 2021

Revised: November 2, 2021

Accepted: November 7, 2021

\section{How to Cite}

Abdul-Hadi Chabuk, H. and Jameel, Z. I. (2021). Relationship between the monoamine oxidase gene overactivity and the other pathophysiological and behavioral parameters implicated in memory deficiency in albino Winstar rats as Alzheimer's disease model. Journal of Applied and Natural Science, 13(4), 1274 - 1282. https://doi.org/10.31018/jans.v13i4.3024

\begin{abstract}
The current study aimed to assess the pathophysiology mechanisms that mediate the effect on albin winstar rats' memory induced by the co -administration of fluoride and aluminum sulfate, as a model of Alzheimer's disease. This was done by assessing monoamine oxidase-A (MAO-A) activity, antioxidant activity, $\mathrm{H}_{2} \mathrm{O}_{2}$ and amyloid- $\beta$ concentration in the hippocampus, embedded deep into the brain's temporal lobe, and level of cytokines in serum. The polymerase chain reaction approach was used to genotyping MAO-A, followed by single -stranded conformational polymorphism (SSCP) coupled with sequencing technique. The experimental animals were divided into two groups: control and treated groups. The uptake of heavy metals led to significantly increased MAO-A activity, amyloid $-\beta$ deposition, $\mathrm{H}_{2} \mathrm{O}_{2}$ and cytokines levels in the treated group. However, the finding showed a significant decrease in antioxidant activity in the treated group. The results indicated that metals caused memory and learning impairments. PCR -SSCP genotyping showed many SNPs and haplotypes of the MAO-A exon 2 region, which showed the MAO-A gene polymorphism changes associated with Alzheimer's disease. The overall results indicated a role of metals to induce oxidative stress stimulating pathophysiological hallmarks in the hippocampus due to an increase in the influx of monoamine oxidase expression, which has been implicated in impaired memory, this study focused on the genetic variation of the exon 2 in monoamine oxidase-A gene and its relationship to Alzheimer's disease with the presence of several single nucleotide polymorphisms that may be related to Alzheimer's disease model in rats.
\end{abstract}

Keywords : Alzheimer's disease, Amyloid - $\beta$. Antioxidant enzyme, Cytokines, MAO-A gene

\section{INTRODUCTION}

Alzheimer's disease (AD) is a serious progressive neurological illness, widely prevalent in the world (Prema et al., 2017). It primarily infects aging men and women and can affect younger people due to industrial development and increased environmental pollution (Zhou et al., 2016). AD is characterized by progressive damage of cognitive domains, learning processes, especially memory loss and behavioral disorders such as mood swings, aggression and social isolation (Hussien et al., 2018). The features of AD that have been reported include stimulating oxidative stress leads to the production of free radicals and neuronal damage, which are considered efficient factors causing behavior and memory disturbances (Zandi et al., 2021). It also con- tributes to the deposition of the extracellular amyloidbeta peptide and the formation of neurofibrillary tangles (NFTs) in the hippocampus (Kalra et al., 2016; Nobakht et al., 2017 and Promyo et al., 2020). Moreover, AD was also shown to induce inflammatory responses through microglial activation and liberation of proinflammatory cytokines such as IL $-1, \mathrm{IL}-12, \mathrm{IL}-6, \mathrm{TNF}-\alpha$, and COX -2 (Abd el-Rady et al., 2021). The monoamine oxidase-A (MAO-A) gene provides guidance for creating a monoamine oxidase- A enzyme. This enzyme is a portion of a family of enzymes that destroy certain molecules called monoamines (neurotransm itters), which convey signals between nerve cells in the brain (Chester et al., 2015). Genetic variations affecting the MAOA gene have been linked to some disorders such as memory deficiency. Some of these genetic 
differences include removing DNA pieces (deletion mutations) from the MAOA gene. Deletion mutations have been detected in individuals with delayed motor skills and mental development. Individuals missing the MAO-A gene have a sharp intellectual disability and difficulty with social interactions (Endo et al., 2014). It has also been observed several common genetic changes(polymorphisms) in or near the MAOA gene affect the activity of the gene increase or decrease (Hajipour et al., 2016). There are studies that suggest that polymorphism of the MAO-A gene and increase activity of the monoamine oxidase-A (MAO-A) enzyme lead to mitochondrial dysfunctions, a synaptic impairment and ion dyshomeostasis, cause dysfunction in the brain implicated in happening Alzheimer's disease and neurological disorders such as senile dementia (Azad et al., 2011 ; Wojtunik-Kulesza et al., 2016 and Abd el $\square$ Rady et al., 2021).

Genetic and environmental factors have been proven to be implicated in AD progression and neurodegenerative diseases. Deposition and abnormal aggregation of heavy metals such as zinc, lead, copper, fluoride, and aluminum major playing role to a contributory in this disease (Justin Thenmozhi et al., 2017), because these metals can be pass through the blood-brain barriers and deposited in different regions of the brain such as the hippocampus and cerebral cortex (Mustafa, 2020). Aluminum sulfate and fluoride are toxicity materials for brain cells. $\mathrm{Al}_{2}\left(\mathrm{SO}_{4}\right)_{3}$ is the material used as a coagulant in drinking water treatment and purification in many areas of the world (Abd El-Rahman, 2003), while fluoride is overlapping industrial pollutants, some foods (such as sardines), pesticides, dietary supplements, and residues (Grandjean, 2019).

The hippocampus region is a limbic structure associated with memory and learning and the site of memory and cognitive disorders initiation as a model of Alzheimer's disease in both humans and rodents. Therefore, aggregation of the heavy metals in this region affects memory and learning (Baranauskaite et al., 2020). This study aimed to assess the likely correlation between exposure to toxicity of heavy metals (by coadministration of fluoride and aluminum) and pathological mechanisms in hippocampus of Winstar rats. It was carried out through the investigation of the activity of the monoamine oxidase-A (MAO- A) enzyme which is related to polymorphism of MAO-A gene, the concentration of amyloid - $\beta$, levels of inflammatory cytokines and $\mathrm{H}_{2} \mathrm{O}_{2}$, and activity of the antioxidant enzymes, all these linked to memory deficits in rats as a model of Alzheimer's disease.

\section{MATERIALS AND METHODS}

\section{Ethics approval}

Experiment protocols were approved by the Depart- ment of Biology, College of Sciences, University of Babylon(Protocol No. 516 /18-May- 2020). According to the National Committee for Research Ethics in Science and Technology (NETNT) (https://www.etikkom.no/en/ ethical-guidelines-for-research/ethical-guidelines-forthe-use-of-animals-in-research/). Written approval was obtained from all participators before study participation.

\section{Experimental animals}

Fifty adult male albino Wistar rats were used obtained from the animal house of the Department of Biology, University of Babylon, Iraq. Animals were kept in a standard condition of temperature and humidity with 12 -h dark-light cycle, and with free access to water and food for two weeks. The male rats were housed four per cage.

\section{Alzheimer's disease model}

This model was designed by exposure of rats to a mixture of aluminum sulfate $\left(\mathrm{Al}_{2}\left(\mathrm{SO}_{4}\right)_{3}\right)$ and fluoride. This model simulates environmental Alzheimer's disease. The metals were obtained from the laboratories of the Department of Chemistry, College of Science, University of Babylon. Dilute mixture solutions of aluminum sulfate and fluoride were prepared (150) $\mathrm{mg} / \mathrm{kg}$ and (20) $\mathrm{mg} / \mathrm{kg}$ body weight, respectively, based on knowledge of the recorded half-lethal dose (LD50) (Han and Choi, 2009). The animals were randomly divided into two main groups. The first group (15 male rats weighing 200-240 $\mathrm{g}$ as a control) were orally administered $1 \mathrm{ml}$ of distilled water for three consecutive months. The second group (35 male rats weighing 200-235 g as treated group) were orally administered a mixture of aluminum and fluoride (150 mg/kg and $20 \mathrm{mg} / \mathrm{kg}$, respectively) for three consecutive months.

\section{Samples preparation and biochemical analysis}

\section{Preparation of tissues}

After the end period of the experiment, the rats were anesthetized with chloroform, the blood samples were collected and divided into two parts. The first part was used for genetic studies, and the second part was used for estimating IL -6 and TNF $-\alpha$ in the serum. The hippocampal cortex was quickly isolated from animals and immediately stored at $-20^{\circ} \mathrm{C}$. Each sample was homogenized (2\% M -pH $7.0-7.2)$ in the ice-cold phosphate buffer solution (PBS) by homogenizer (Potter-Elvehjem Tissue Grinder consist of PTFE smooth pestle for soft tissues, china). The hippocampal tissue homogenates were centrifuged at $4{ }^{\circ} \mathrm{C}$. and $1500 \mathrm{~g}$ for $20 \mathrm{~min}$. and the supernatants were used to estimate the activity of monoamine oxidase, antioxidants activity, $\mathrm{H}_{2} \mathrm{O}_{2}$ level and amyloid $\beta$ concentration. 
Monoamine oxidase-A (MAO-A) activity analysis

The activity of the MAO-A enzyme was determined using Kit (Fluorometric) manufactured by (Arigo Biolaboratories Corporation/Taiwan). "Principle of the test provides a convenient fluorimetric suitable means for measure MAO-A enzyme activity. In the test, MAOA reacts with $p$-tyramine, resulting in $\mathrm{H}_{2} \mathrm{O}_{2}$ formation of, which is determined by a fluorimetric method ( $\mathrm{\lambda em} /$ $\lambda e x=585 / 530 n m) "$. Measure the fluorescence (FLU) of the samples and standards at $\lambda e x=530 / \lambda$ em $=585$ $\mathrm{nm}$.

MAO enzyme activity is calculated in the samples:

$$
\text { MAO Activity (units/g) }=\frac{\text { FLU sample }- \text { FLU control }}{\text { Slope } \times \mathrm{t}}
$$

FLU sample $=$ Absorbance measure in an unknown samples. FLU control = Absorbance measure in control samples (samples in presence of inhibitor). Slope = Determine from the calibration curve $(\mu \mathrm{M}-1) . t=$ incubation time (20 minutes).

\section{Estimation of the inflammatory cytokines (TNF - $\alpha$ and IL -6) \\ The tumor necrosis factor Alpha (TNF - $\alpha$ ) and Interleu- kin -6 (IL -6) levels ( $\mathrm{pg} / \mathrm{ml}$ ) in rats serum were evaluat- ed using an ELISA kit from (Elabscience, China) follow- ing the manufacturer's protocol. The absorbance was read at $450 \mathrm{~nm}$ that is uses for the immunoassay tech- niques of the quantitative sandwich enzymes.}

\section{Determination activity of the antioxidant enzymes} The activity of the catalase (CAT), glutathione $S$ transferase (CAT), and superoxide dismutase (SOD) enzymes were analyzed in hippocampal tissue using an ELISA kit from (Elabscience, China).

\section{Estimation of amyloid- $\beta(A \beta)$ and hydrogen perox-} ide $\left(\mathrm{H}_{2} \mathrm{O}_{2}\right)$ level in hippocampus

The $A \beta$ concentration was determined using the Sandwich ELISA kit principle and $\mathrm{H}_{2} \mathrm{O}_{2}$ level was measured using the fluorescence assay Kit according to the manufacturer's instructions (Elabscience, China).

\section{Behavioral analysis}

The behavioral tests were measured near the experiment's end period to assess the effect of heavy metals that induced Alzheimer's disease on the memory and cognition of the rats by the radial arm maze test (RAM) according to the method of Justin Thenmozhi et al. (2017). The following parameters such as working memory and reference memory, were determined. The working memory is a calculation of the numbers of duplicated entries to baited arms, indicated as (short term memory) and reference memory is the calculation of the number of entries to unbaited arms, indicated as the knowledge required for a particular situation that remains constant over time (long -term memory). The time required to complete the test in all experiments was calculated. All the behavioral tests were achieved from 09.00 a.m. to 12.00 p.m. for $10 \mathrm{~min}$ for three days.

\section{Genetic analysis}

Animals were sacrificed with chloroform anesthetic, blood samples were collected from the left ventricle by needle stab and $2 \mathrm{ml}$ of blood was put in the EDTA tubes. Genomic DNA was obtained from the white blood cells of both the treatment and control groups were extracted using a DNA extraction kit (Favorgen). The PCR -SSCP approach was examined after the target site for MAO-A genotyping was expanded. The specific primer used during the present study was designed.

\section{PCR primer design.}

PCR primer pair of rat monoamine oxidase-A (MAO-A) gene was designed by the NCBI primer BLAST server to consolidate the targeted SNPs in the designed fragments that are localized in MAO-A exon 2 region haplotypes dependent on Primer3 plus MAO-A gene ID reference sequences: NC -00 5120.4. The genetic fragments length of (120 bp) was made to set the recommended amplicon length in the PCR -SSCP protocol (Hashim \& Al-Shuhaib, 2019). The sequence forward and reverse primer was Forward 5'CATCCCACCGATTTTGACTGC3', Reverse5'TCCTTGTAATACACCATGCACT3'.

\section{Statistical Analysis}

Statistical significance was measured by analysis by an independent $t$-test between two groups. The value of $p<0.05$ was considered to be statistically significant for all exams. Analysis correlation between parameters done by Pearson's test (SPSS version 23).

\section{RESULTS}

\section{Biochemical analysis}

Fig. 1(A, C, D) show that the activity of the MAO-A enzyme, $\mathrm{H}_{2} \mathrm{O}_{2}$ level and amyloid $-\beta$ concentration in the hippocampus of the experimental group were significantly $(P<0.05)$ increased compared to the control group. The activities of antioxidant enzymes (SOD, CAT and GSH) were significantly $(P<0.05)$ decreased in the hippocampus of the treated rat's group as compared to the control group (Fig. 1B). Long-term exposure to metals in the rats of the treated group significantly $(P<0.05)$ increased the generation of serum proinflammatory cytokines such as IL -6 and TNF - $\alpha$ compared to the control group, Fig. 1(E). 
(A)

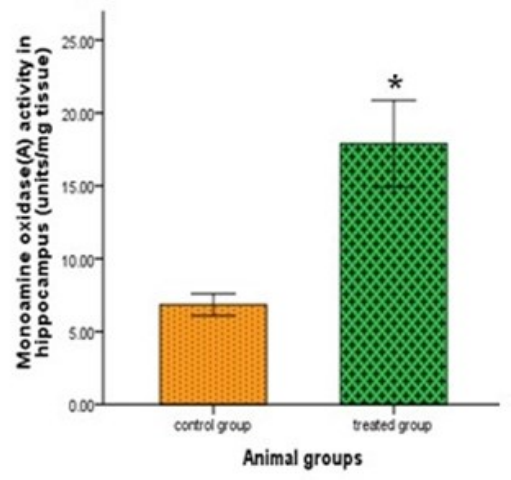

(D)

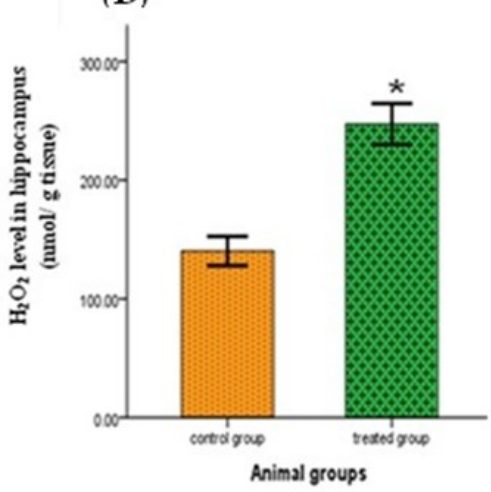

(B)

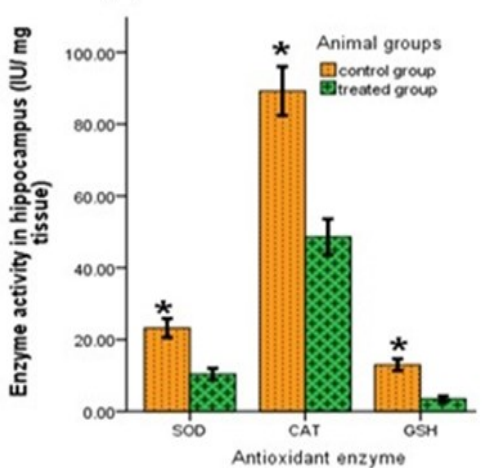

c)

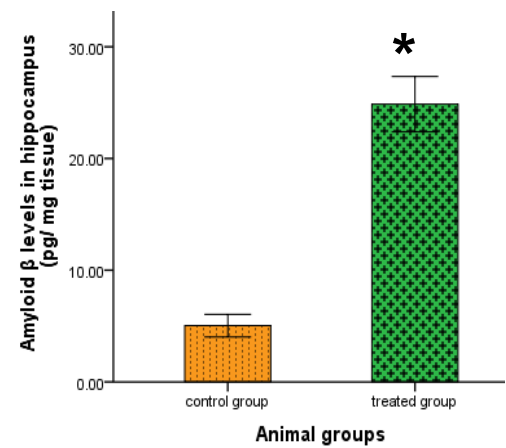

(E)

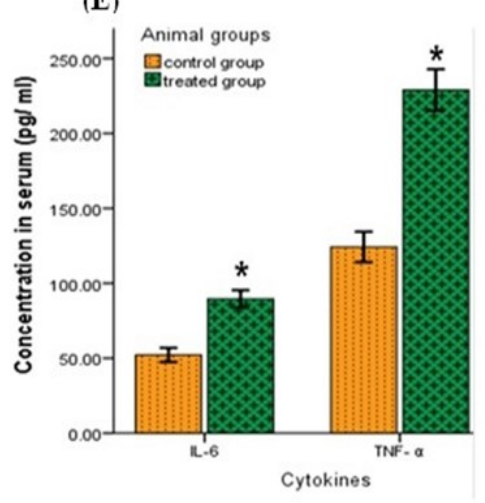

Fig. 1. Showing changes in the activity of MAO-A (A), antioxidant enzymes (catalase (CAT), glutathione S-transferase $(\mathrm{GSH})$, superoxide dismutase $(\mathrm{SOD})$ ) $(B)$, concentrations of amyloid- $\beta(\boldsymbol{C})$, and hydrogen peroxide level $\left(\mathrm{H}_{2} \mathrm{O}_{2}\right)(\boldsymbol{D})$ in hippocampal tissue, and serum inflammatory cytokines levels (tumor necrosis factor- $\alpha$ (TNF- $\alpha$ ) and interleukin-6 (IL-6)) $(E)$ in the rats treated by a mixture of aluminum and fluoride (as model Alzheimer's disease in the rats) for 3 months. Data were represented as $M \pm$ S.E. $(*)$ represent significantly different at $(p<0.05)$ between the control group $(n=15)$ and treated group $(n=35)$.

\section{Behavioral analysis}

The analysis of the radial arm maze tests data, the rats treated with a mixture of aluminum and fluoride exhibited more errors in both the working memory and reference memory tests and needed more time to complete this task as compared to the control group, Fig. 2.

Correlations between MAO-A enzyme and the other pathophysiological parameters of Alzheimer's disease:

The major pathological features of Alzheimer's disease involve not only amyloid $-\beta$ plaques deposition but also stimulation oxidative stress, altered activities of antioxidants, and production of proinflammatory cytokines. These pathological features have complex interactions with the increased activity of MAO-A. Table (1) shows the relationship of pathological parameters (IL6, TNF, SOD, CAT, GSH, $\mathrm{H}_{2} \mathrm{O}_{2}$, and amyloid $-\beta$ ) with MAO-A. There were positively significant relationships of IL -6 , TNF- $\alpha, \mathrm{H}_{2} \mathrm{O}_{2}$ and amyloid $-\beta$ with the MAO-A enzyme. The antioxidant enzymes (CAT, GSH and CAT) were negatively related to the MAO-A enzyme. There were positively significant relationships between memory test (working, reference) and activity of MAO-A enzyme.

Table 1. Correlations between the MAO-A enzyme overactivity and the other pathophysiological and behavioral parameters of Alzheimer's disease, $\mathrm{n}=50$.

\begin{tabular}{|c|c|c|c|c|c|c|c|c|c|c|}
\hline \multicolumn{2}{|c|}{ Biochemical } & \multirow{2}{*}{$\frac{\text { IL-6 }}{0.373^{* *}}$} & \multirow{2}{*}{$\frac{\text { TNF- } \alpha}{0.463^{* *}}$} & \multirow{2}{*}{$\begin{array}{l}\text { SOD } \\
-0.422^{* *}\end{array}$} & \multirow{2}{*}{$\begin{array}{l}\text { CAT } \\
-0.617^{\star *}\end{array}$} & \multirow{2}{*}{$\begin{array}{l}\text { GSH } \\
-0.531^{* \star}\end{array}$} & \multirow{2}{*}{$\frac{\mathrm{H}_{\mathbf{2}} \mathrm{O}_{\mathbf{2}}}{0.463^{\text {** }}}$} & \multirow{2}{*}{$\begin{array}{l}\text { Amyloid- } \beta \\
0.814^{* \star}\end{array}$} & \multirow{2}{*}{ 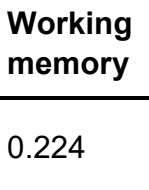 } & \multirow{2}{*}{$\begin{array}{l}\begin{array}{l}\text { Reference } \\
\text { memory }\end{array} \\
0.188\end{array}$} \\
\hline \multirow{2}{*}{ MAO-A } & $\begin{array}{l}\text { Pearson } \\
\text { Correlation }\end{array}$ & & & & & & & & & \\
\hline & $\begin{array}{l}\text { Sig. } \\
\text { (2-tailed) }\end{array}$ & 0.008 & 0.001 & 0.002 & 0.000 & 0.000 & 0.001 & 0.000 & 0.118 & 0.191 \\
\hline
\end{tabular}

${ }^{* *}$ Correlation is significant at the 0.01 level (2 -tailed). 



Fig. 2. Showing effect of the exposure of heavy metals for 3 weeks on learning and memory abilities of rats (as model of Alzheimer's disease in the rats) by radial arm maze tests.(A) Showing evaluation of both the reference and working memories. (B) Showing the time required by rats to end to maze,Data is represented as $M \pm S$.E. ; (*) represent significantly different at $p<0.05$ between control group $(n=15)$ and treated group $(n=35)$.

\section{MAO-A gene}

Fig. 3 shows agarose gel electrophoresis of an amplified product patterns of MAO-A gene (120bp) for treatment and control groups, Fig. 4 shows PCR-SSCP for momamine oxidase-A gene. The results indicated that there was the existence of two different conformational haplotypes in treated groups, including the 1st haplotype with two bands, the 2nd haplotype patterns with 3 bandsm, table (2). These DNA polymorphisms must therefore be verified by in silico and DNA sequence, Fig. 5.

Fig. 6 position of methylated cytosines in CpG occurrence in the seguences of the MAO-A gene shows that these seguences contained 30 methylated CpG- dinucleotides.

\section{DISCUSSION}

Aluminum and fluoride are among the heavy metals that cause neurotoxicity through several mechanisms that lead to changes in the behavioral and biochemical parameters (Hussien et al., 2018). The results in Fig. 1. indicated that MAO-A enzyme overactivity, amyloid - $\beta$ deposition, inhibition of antioxidants enzymes in the hippocampal brain of treated rats, and activated inflammatory cytokines play important roles in inducing the pathophysiology of Alzheimer's disease. In addition to the significant elevations in $\mathrm{H}_{2} \mathrm{O}_{2}$, which mainly lead to increased oxidative stresses in the treated group, these results are consistent with (Xie et al., 2015 ; WojtunikKulesza et al., 2016 ; Hussien et al., 2018 and Grandjean 2019). Accumulation of fluoride in the brain causes neurotoxicity through changes in the nucleic acid metabolism and proteolytic enzymes that cause DNA damage may be due to these metals create oxidative stress and free radicals in the hippocampus responsible for neuronal destruction and death, DNA damage and mitochondrial dysfunctions (Kalra et al.,
2016). Oxidative stress plays a critical role in memory disorders induces the development of ageing and Alzheimer's disease due to the imbalance between the removal and production of reactive oxygen species (ROS) (Weinreb et al., 2016). The free radical may be targeting the cellular proteins, polysaccharides and lipids, and increases the activities of the mitochondrial enzymes MAO-A which catalyze the oxidative deamination of many amines, such as monoamine neurotransmitters (dopamine, serotonin, and noradrenaline), resulting in a decrease their levels in the hippocampus which are linked to the progression of Alzheimer's disease and memory deficits (Naoi et al., 2016).

A further increase in oxidative stress in the hippocampus may arise from the raised activities of the mitochondrial enzymes MAO-A. This enzyme is associated with neurodegeneration through oxidative stress (derived from the increased formulation of hydrogen peroxide) (Weinreb et al., 2016). The activated MAO-A enzyme raises the expression of g-secretase and bsecretase and improves $A \beta$ formation from amyloid precursor proteins (APP). Activated MAO-A could also be connected with the production of neurofibrillary tangles (Zhou et al., 2016).

In general, the significantly increased hydrogen peroxide $\left(\mathrm{H}_{2} \mathrm{O}_{2}\right)$ concentrations are inactivated by the $\mathrm{Fe} 2+$ ions and glutathione peroxidase (GPO). This increase in hydrogen peroxide was accompanied by a significant decrease in activities of antioxidant enzymes in the rat's brain, which is considered the first line of defence against the free radicals attack, and a decrease in their activities can contribute to the tissues damage (Kumar et al., 2017). Oxidative stress stimulating inflammatory cytokines (IL- 6 , TNF- $\alpha$ ) and amyloid fragments may be due to a decrease in efflux neurotransmitters expression and an increase in influx monoamine oxidase expression, leading to a rising concentration of $A \beta$ in the brain parenchyma, Table 1 (Church et al., 2014 and 


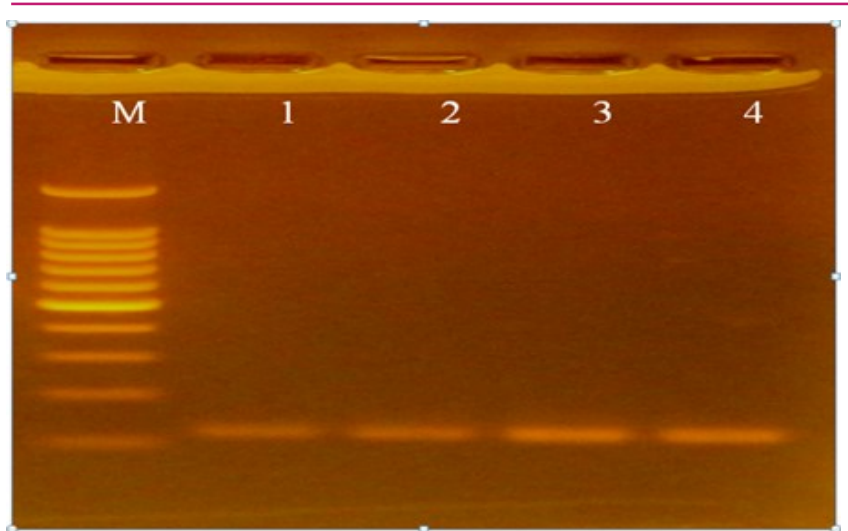

Fig. 3. Agarose gel electrophoresis of an amplified product patterns of MAO-A gene (120bp) for treatment and control groups

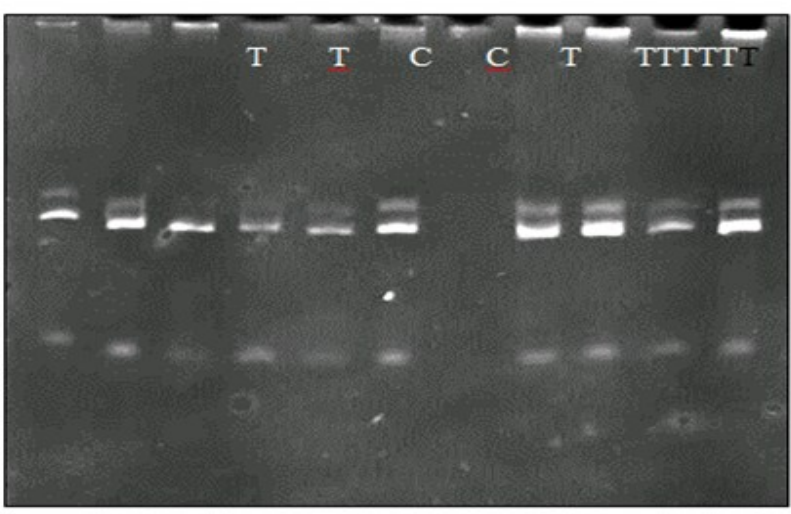

Fig. 4. PCR-SSCP for MAO-A gene. Lanes 1-10 refer to MAO-A gene PCR-SSCP fragment patterns (T treatment , $C$ control) Electrophoresis condition: $8 \%$ polyacrylamide gel concentration; $200 \mathrm{~V}(7.5 \mathrm{~V} / \mathrm{cm})-100 \mathrm{~mA}$, run time 90 - 120 min. Staining method; ethidium bromide

Hung et al., 2016). This study exhibits that amyloid- $\beta$ accumulation is associated with markedly of behavioral alters and neurogenesis defect.

Reference memory and working memory are estimated in rats by using radial arm maze tests (Fig. 2.). Working memory is estimated by taking into account the rat's entry into each arm once, and the reference memory is examined the number of times the rat enters the arms containing food. If the rats re-enter in the arms or fail to enter into the arm that is food-containing, it is considered a reference and working memory errors (Prema et al., 2017). In the present results, treatment with aluminum and fluoride decreased the working memory and reference memory in the radial arm maze tests that were implicated in the disruption of memory related functions due to the accumulation of heavy metals in the hippocampal region that led to memory impairment and neurodegenerative disorders including neuronal loss, cognitive deficits, and learning deficits. CHabuk et al. (2019) showed that aluminum chloride caused decrease levels of brain neurotransmitters and inhibited avoidance latency and escape latency in the elevated plus-maze tests indicating memory impairment in rats. This study suggested that heavy metals cause MAO-A overactivity in the hippocampus, which has been associated with cognitive and learning deficits.

The current sequence results in Fig.5. showed several SNPs in different positions in the exon region ( $G 41 \mathrm{~A}$, T 46 A, C 47 T, C 48 T, A 49 C, C50 A, T 52 C, G 53 T, A 54 G, G 55 A, A 56 A, G57 A, A 58 G, A 67 T, A 68 C, T 69 C, C 70 A, C 71 G, A 72 C, G 73 C, C 74 G, G 75 T, T 76 C , T 78 C, C 80 T ). Polymorphisms in the MAO-A gene have also been identified and these variants have been associated with psychiatric or behavioral phenotypes. These variants affect the MAO-A functions, which are associated with higher levels of MAO-A activity. This study is in line with several studies showing that genetic variations in MAO-A cause alterations in the functions of brain neurotransmitters, including metabolism, enzymes activity, and reception in different brain regions, resulting in an imbalance in the neurotransmitter systems (Kudryavtseva, 2006). While another study showed that the physiological regulation of the MAO-A gene expression, the hormonal and neurochemical regulation of which has been investigated in sufficient detail, can be explored in rats with hyperactivity behavior patterns (Ul'yana et al., 2013).

The interactions between 5 HTT and MAO-A polymorphisms have an impact on the anterior cortex of brain region activities that have been associated with impulsivity, such as impulsive violence (Passamonti et al., 2008). Polymorphism in MAO-A can also damage immune functions, increase agonistic behavior, and trigger hyper -aroused behaviors (Toth et al., 2012). Another study showed that the MAO-A polymorphism is associated with anxiety, depression, impulsivity and memory deficits (Sjöberg et al., 2006). The relationship between the genetic variability of this gene and these traits has not been consistent. Both interactions with environmental factors such as heavy metal exposure

Table 2. PCR-SSCP haplotype distribution of MAO-A gene

\begin{tabular}{|c|c|c|c|c|}
\hline Genotype & Treated group & Control group & P Value & OR=(95\%Cl) \\
\hline 2 bands & $1(2.8 \%)$ & $5(33.3 \%)$ & & \\
\hline 3 bands & $34(97.2 \%)$ & $10(66.6 \%)$ & $0.007^{*}$ & $0.05(0.006-0.56)$ \\
\hline total & 35 & 15 & & \\
\hline
\end{tabular}

$\mathrm{P} \leq 0.05 ; \mathrm{OR}=(95 \% \mathrm{Cl})$. 


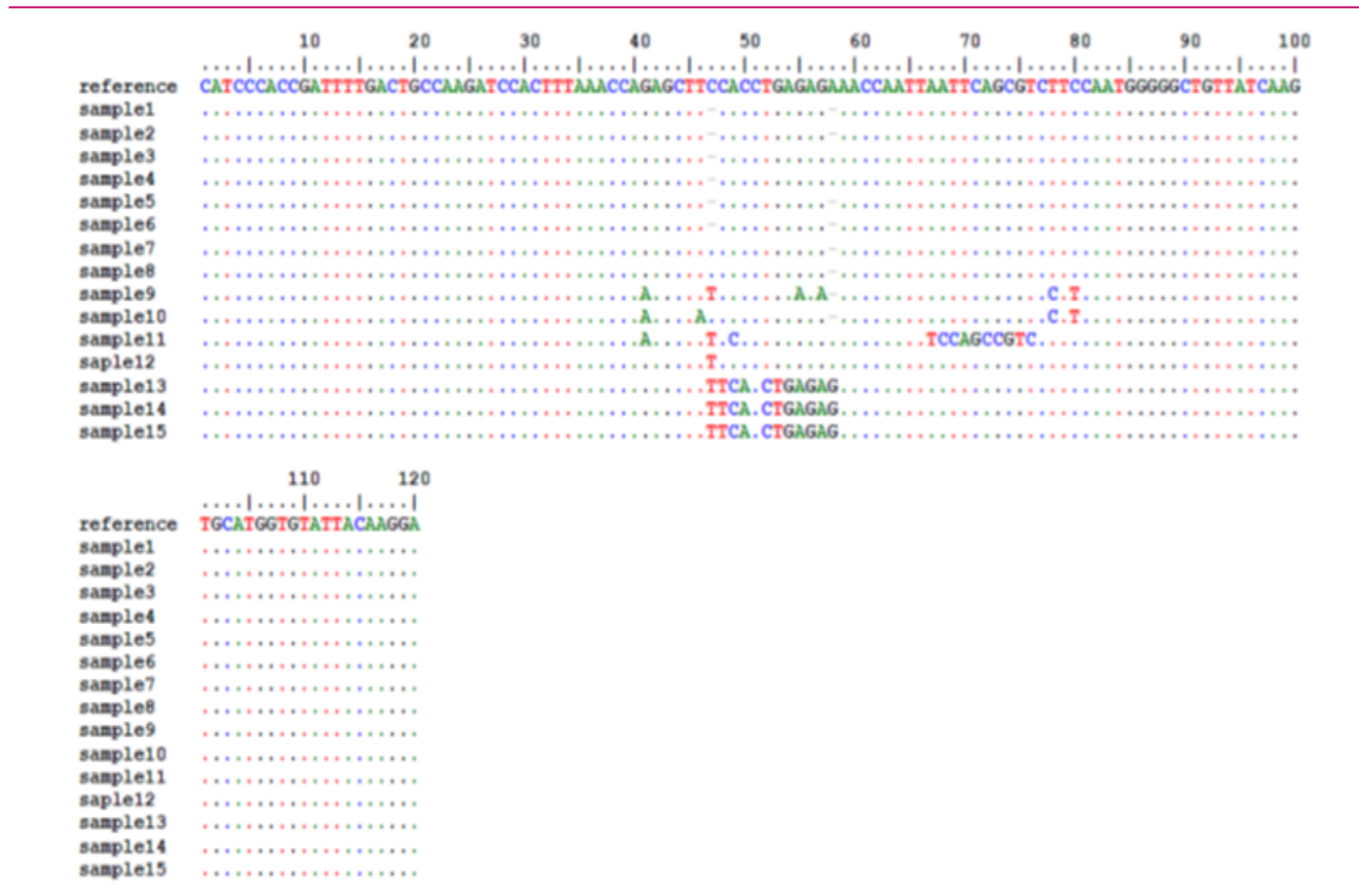

Fig.5. Sequence alignment of exon 2 region MAO-A gene accession number NC-005120.4 by Bio Edit program version 7.2 .5

In total 15 sequences have been analyzed.

Position of methylated cytosines in $\mathrm{CpG}$ context in the alignment

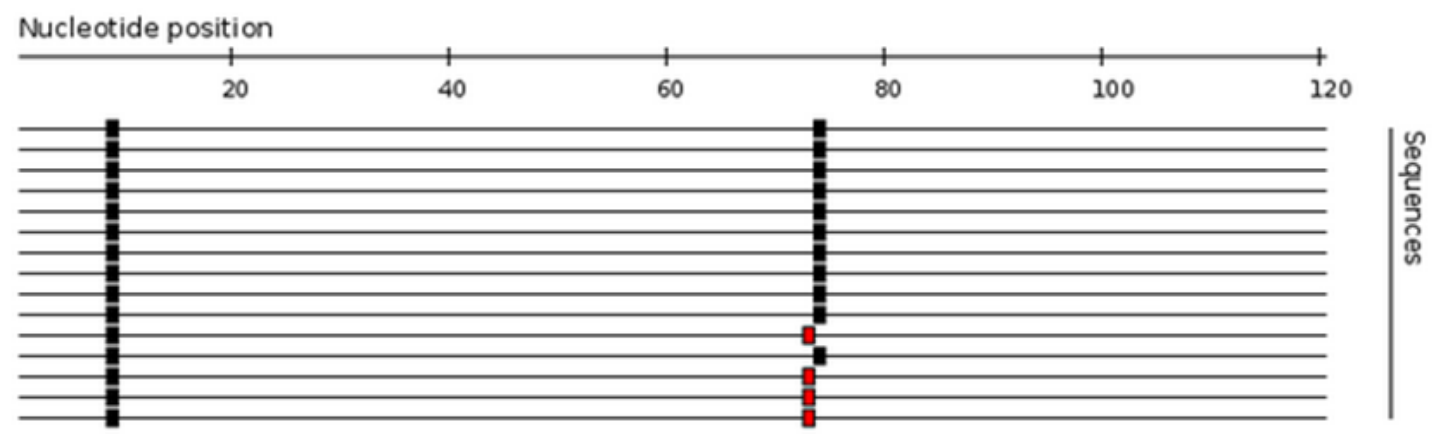

\section{Methylation distribution among the repetitive sequences:}

\section{Statistics about the methylated CpG occurrence in the sequences:}

In total these sequences contained $\mathbf{3 0}$ methylated $\mathrm{CpG}$-dinucleotides.

On average 2.00 CpG-dinucleotides have been found in each DNA Sequence corresponding to $\mathbf{1 . 6 7}$ methylated Cytosines in 100 nucleotides.

Fig. 6. MAO-A gene methylation 
have been shown (Lau et al., 2009).

Sequence analysis of MAO-A showed the presence of two $\mathrm{CpG}$ islands in the gene (Fig. 4.). The first CpG island is located in 9 , and the second $\mathrm{CpG}$ island is located in 74. Methylation (Fig. 6.) can be detected and quantified by many methods, but all studies currently available have certain advantages. We have therefore tried to assess the region's epigenetic potential using sequence-based analysis systematically. In fact, all bioinformatics analysis has its own features. Such as one advantage of the in silico analysis is that it is independent of the detection methods used. The sequencebased analysis is not dependent on the type of biological sample nature used for the analysis (Bock et al., 2007). Genetic variations and epigenetic variants of the MAO-A can be a solid indicator of the enzyme's catalytic activity in question, possibly due to a variety of environmental factors. Nevertheless, this result can be viewed as an even more accurate and precise biological index for neurodegenerative disorders (Godar et al., 2016).

\section{Conclusion}

The current study obviously points to the role of heavy metals in inducing oxidative stress, the inhibition of antioxidant activity, the hyperactivity of the MAO-A enzyme linked with revealing the gene polymorphism MAO-A changes, and the amyloid- $\beta$ deposition in the hippocampal tissues of the rats, which were associated with memory deficits in addition to stimulating inflammatory cytokines. These factors played a critical role in inducing the pathophysiology of the Alzheimer's disease model in adult albino Winstar rats.

\section{ACKNOWLEDGEMENTS}

The authors would like to thank the College of Science, the University of Babylon, for providing the necessary facilities during this study.

\section{Conflict of interest}

The authors declare that they have no conflict of interest.

\section{REFERENCES}

1. Abd El-Rahman, S. S. (2003). Neuropathology of aluminum toxicity in rats (glutamate and GABA impairment). Pharmacological Research, 47(3), 189-194.

2. Abd el $\square$ Rady, N. M., Ahmed, A., Abdel-Rady, M. M., \& Ismail, O. I. (2021). Glucagon-like peptide-1 analog improves neuronal and behavioral impairment and promotes neuroprotection in a rat model of aluminum-induced dementia. Physiological Reports, 8(24), e14651.

3. Azad, N., Rasoolijazi, H., Joghataie, M. T., \& Soleimani, S. (2011). Neuroprotective effects of carnosic acid in an experimental model of Alzheimer's disease in rats. Cell Journal (Yakhteh), 13(1), 39.

4. Baranauskaite, J., Sadauskiene, I., Liekis, A., Kasauskas, A., Lazauskas, R., Zlabiene, U., Bernatoniene, J. (2020). Natural Compounds Rosmarinic Acid and Carvacrol Counteract Aluminium-Induced Oxidative Stress. Molecules, 25(8), 1807.

5. Bock, C., Walter, J., Paulsen, M., \& Lengauer, T. (2007). CpG island mapping by epigenome prediction. PLoS Comput Biol, 3(6), e110.

6. CHabuk, H. A-H., Al-Harbi, H. J., \& Al-Saadi, H. K. Z. (2019). Aluminum chloride administration Induced behavioral and physiological changes in adult male rats. Indian Journal of Public Health Research \& Development, 10 (10).

7. Chester, D. S., DeWall, C. N., Derefinko, K. J., Estus, S., Peters, J. R., Lynam, D. R., \& Jiang, Y. (2015). Monoamine oxidase $A$ (MAOA) genotype predicts greater aggression through impulsive reactivity to negative affect. Behavioural Brain Research, 283, 97-101.

8. Church, R. M., Miller, M. C., Freestone, D., Chiu, C., Osgood, D. P., Machan, J. T., Silverberg, G. D. (2014). Amyloid-beta accumulation, neurogenesis, behavior, and the age of rats. Behavioral Neuroscience, 128(4), 523.

9. Endo, Y., Saito, Y., Otsuki, T., Takahashi, A., Nakata, Y., Okada, K., Takada, E. (2014). Persistent verbal and behavioral deficits after resection of the left supplementary motor area in epilepsy surgery. Brain and Development, 36(1), 74-79.

10. Godar, S. C., Fite, P. J., McFarlin, K. M., \& Bortolato, M. (2016). The role of monoamine oxidase $A$ in aggression: Current translational developments and future challenges. Progress in Neuro-Psychopharmacology and Biological Psychiatry, 69, 90-100.

11. Grandjean, P. (2019). Developmental fluoride neurotoxicity: an updated review. Environmental Health, 18(1), 1-17.

12. Hajipour, S., Sarkaki, A., Farbood, Y., Eidi, A., Mortazavi, P., \& Valizadeh, Z. (2016). Effect of gallic acid on dementia type of Alzheimer disease in rats: electrophysiological and histological studies. Basic and Clinical Neuroscience, 7(2), 97.

13. Han, S.-H., \& Choi, D.-H. (2009). Effects of aluminum feedings on aluminum, phospholipid and catecholamine concentrations in old rat brain tissue. Journal of the Korean Society of Food Culture, 24(2), 236-243.

14. Hashim, H. O., \& Al-Shuhaib, M. B. S. (2019). Exploring the potential and limitations of PCR-RFLP and PCRSSCP for SNP detection: A review. Journal of Applied Biotechnology Reports, 6(4), 137-144.

15. Hung, A. S. M., Liang, Y., Chow, T. C. H., Tang, H. C., Wu, S. L. Y., Wai, M. S. M., \& Yew, D. T. (2016). Mutated tau, amyloid and neuroinflammation in Alzheimer disease-A brief review. Progress in Histochemistry and Cytochemistry, 51(1), 1-8.

16. Hussien, H. M., Abd-Elmegied, A., Ghareeb, D. A., Hafez, H. S., Ahmed, H. E. A., \& Abd El-moneam, N. (2018). Neuroprotective effect of berberine against environmental heavy metals-induced neurotoxicity and Alzheimer's-like disease in rats. Food and Chemical Toxicology, 111, 432444.

17. Justin Thenmozhi, A., William Raja, T. R., Manivasagam, T., Janakiraman, U., \& Essa, M. M. (2017). Hesperidin 
ameliorates cognitive dysfunction, oxidative stress and apoptosis against aluminium chloride induced rat model of Alzheimer's disease. Nutritional Neuroscience, 20(6), 360 -368 .

18. Kalra, J., Kumar, P., Majeed, A. B. A., \& Prakash, A. (2016). Modulation of LOX and COX pathways via inhibition of amyloidogenesis contributes to mitoprotection against $\beta$-amyloid oligomer-induced toxicity in an animal model of Alzheimer's disease in rats. Pharmacology Biochemistry and Behavior, 146, 1-12.

19. Kudryavtseva, N. N. (2006). The Psychopathology of Repeated Aggression: A Neurobiological Aspect.

20. Kumar, B., Prakash Gupta, V., \& Kumar, V. (2017). A perspective on monoamine oxidase enzyme as drug target: challenges and opportunities. Current Drug Targets, 18(1), 87-97.

21. Lau, J. Y. F., Goldman, D., Buzas, B., Fromm, S. J., Guyer, A. E., Hodgkinson, C.,\& Pine, D. S. (2009). Amygdala function and 5-HTT gene variants in adolescent anxiety and major depressive disorder. Biological Psychiatry, 65 (4), 349-355.

22. Mustafa, H. N. (2020). Neuro-amelioration of cinnamaldehyde in aluminum-induced Alzheimer's disease rat model. Journal of Histotechnology, 43(1), 11-20.

23. Naoi, M., Riederer, P., \& Maruyama, W. (2016). Modulation of monoamine oxidase (MAO) expression in neuropsychiatric disorders: genetic and environmental factors involved in type A MAO expression. Journal of Neural Transmission, 123(2), 91-106.

24. Nobakht, M., Hoseini, S. M., Mortazavi, P., Sohrabi, I., Esmailzade, B., Roosh, N. R., \& Omidzahir, S. (2011). Neuropathological changes in brain cortex and hippocampus in a rat model of Alzheimer's disease. Iranian Biomedical Journal, 15(1-2), 51.

25. Passamonti, L., Cerasa, A., Gioia, M. C., Magariello, A., Muglia, M., Quattrone, A., \& Fera, F. (2008). Genetically dependent modulation of serotonergic inactivation in the human prefrontal cortex. Neuroimage, 40(3), 1264-1273.

26. Prema, A., Justin Thenmozhi, A., Manivasagam, T., Mohamed Essa, M., \& Guillemin, G. J. (2017). Fenugreek seed powder attenuated aluminum chloride-induced tau pathology, oxidative stress, and inflammation in a rat model of Alzheimer's disease. Journal of Alzheimer's Disease, 60(s1), S209-S220.

27. Promyo, K., Iqbal, F., Chaidee, N., \& Chetsawang, B. (2020). Aluminum chloride-induced amyloid $\beta$ accumulation and endoplasmic reticulum stress in rat brain are averted by melatonin. Food and Chemical Toxicology, 146,111829

28. Sjöberg, R. L., Nilsson, K. W., Nordquist, N., Öhrvik, J., Leppert, J., Lindström, L., \& Oreland, L. (2006). Development of depression: sex and the interaction between environment and a promoter polymorphism of the serotonin transporter gene. International Journal of Neuropsychopharmacology, 9(4), 443-449.

29. Toth, M., Tulogdi, A., Biro, L., Soros, P., Mikics, E., \& Haller, J. (2012). The neural background of hyper-emotional aggression induced by post-weaning social isolation. Behavioural Brain Research, 233(1), 120-129.

30. Ul'yana, A. B., Bondar, N. P., Filipenko, M. L., \& Kudryavtseva, N. N. (2013). Downregulation of serotonergic gene expression in the Raphe nuclei of the midbrain under chronic social defeat stress in male mice. Molecular Neurobiology, 48(1), 13-21.

31. Weinreb, O., Amit, T., Bar $\square$ Am, O., \& Youdim, M. B. H. (2016). Weinreb. British Journal of Pharmacology, 173 (13), 2080-2094.

32. Wojtunik-Kulesza, K. A., Oniszczuk, A., Oniszczuk, T., \& Waksmundzka-Hajnos, M. (2016). The influence of common free radicals and antioxidants on development of Alzheimer's Disease. Biomedicine \& Pharmacotherapy, 78, 39-49.

33. Xie, S., Chen, J., Li, X., Su, T., Wang, Y., Wang, Z., ... Li, $X$. (2015). Synthesis and evaluation of selegiline derivatives as monoamine oxidase inhibitor, antioxidant and metal chelator against Alzheimer's disease. Bioorganic \& Medicinal Chemistry, 23(13), 3722-3729.

34. Zandi, N., Pazoki, B., Roudsari, N. M., Lashgari, N.-A., Jamshidi, V., Momtaz, S., Akhondzadeh, S. (2021). Prospects of Saffron and its derivatives in Alzheimer's disease. Archives of Iranian Medicine (AIM), 24(3).

35. Zhou, X., Li, Y., Shi, X., \& Ma, C. (2016). An overview on therapeutics attenuating amyloid $\beta$ level in Alzheimer's disease: targeting neurotransmission, inflammation, oxidative stress and enhanced cholesterol levels. American Journal of Translational Research, 8(2), 246. 